\title{
Sabine Berghahn Ehe und Familie in der Verfassungsdiskussion - vom institutionellen zum sozialen Grundrechtsverständnis?
}

Viel ist nicht herausgekommen. Die neue Verfassungsdebatte, dic von bürgerrechtlichen und frauenpolitischen Initiativen angestoßen so hoffnungsvoll begonnen hatte, ist durch den Einigungsvertrag und dic institutionelle Kanalisierung in der Gemeinsamen Verfassungskommission von Bundestag und Bundesrat schnell zu einer Alibi-Veranstaltung, einem parteicnpolitischen Macht-Malefiz geworden. In frauenpolitischer Hinsicht hat sie wenigstens eine Kompromißformulierung zur normativen Verstärkung von Art. 3 Abs. 2 Grundgesetz hervorgebracht, welche zwar immer noch nicht eindeutig klärt, ob die bisher übliche milde Quotierung, die in einigen Bundesländern für die Einstellung und Beförderung von Frauen im öffentlichen Dienst gilt, verfassungsgemäß ist oder nicht, die immerhin aber cin Interpretationsindiz zugunsten einer effektiven Gleichstellungsförderung schaffe.

\section{Die Verfassungsreformdiskussion - auch - um Art. 6 Grundgesetz}

Sind aber Gleichberechtigung, Gleichstellung oder gleiche Teilhabe von Frauen an den gesellschaftlichen Ressourcen und Lebenschancen verfassungsrechtlich nur von Art. 3 Grundgesetz determiniert? Ist dort schon alles gesagt, was Einfluß auf die existierende Asymmetrie der Geschlechterverhältnisse ausübt oder ausüben könnte? Die Fragen sind rhetorisch, denn der Hauptgrund für die weiterbestehende Benachteiligung von Frauen bleibt die ungleiche gesellschaftliche Zuschreibung der Verantwortlichkeit für Reproduktionsarbeit, d.h. Kinderbetreuung und Hausarbeit, und die rechtliche Privilegierung des kontinuierlich crwerbstätigen und besser verdienenden Ehepartners, sprich Mannes. Faktisch geht es um Binnenstrukturen von Zweierbeziehungen und Familien, verfassungsrechtlich geht es also auch um Art. 6 Grundgesetz.

In der neuen Verfassungsdebatte haben Frauen schon sehr früh eine Generalrevision des Art. 6 GG gefordert. Anstoß wurde vor allem an Abs. I genommen, der Familie und Ehe unter den "besonderen Schutz" des Staates stellt, obwohl es sich doch hier um zwei sehr verschiedene Sachverhalte handclt, deren - vor allem - soziale Schuzzbedürftigkeit und Förderungswürdigkeit in der Gescllschaft kaum mchr gleich beurteilt wird. Mit anderen Worten: Die Einordnung der Ehe auf derselben Stufe mit der Familie wird als überholte Privilegierung und damit als Benachteiligung nichtehelicher Formen des Zusammenlebens von Erwachsenen (mit Kindern oder ohne) kritisiert und daher für reformbedürftig gehalten. Statt der Ehe soll demnach nur noch die - begrifflich weit verstandene - Familic unabhängig vom rechtlichen Status ihrer Mitglieder, d. h. das Zusammenleben mit Kindern und pflegebedürftigen Menschen besonderen staatlichen Schutz und entsprechende Förderung genießen (vgl. 
Art. 6 Abs. I des Kuratoriumsentwurfs,' ausführlicher s.u.). Als Kompromißlinie gegenüber dem Beharren auf dem besonderen Schutz der Ehe wurde zum Teil formuliert, daß wandere Lebensgemeinschaften" als die Familien, also auch Ehen, mindestens "geachtet oder nvor Diskriminierung geschützt « werden sollten. ${ }^{2}$ Des weiteren wurden die wirksame Förderung der Vereinbarkeit von Beruf und Kindererziehung, Entlastungen und Hilfen für Alleinerziehende, die Garantie öffentlicher Kinderbetreuung und der Ausbau der Rechtsstellung von Kindern einschließlich der Formulierung emanzipatorischer Erziehungsziele und gewaltfreier Methoden gefordert.

Wen wundert's angesichts der Fülle von grundlegenden Akzentverschiebungen, daß in der Gemeinsamen Verfassungskommission von Bundestag und Bundesrat, in der übrigens Frauen mit einem noch geringeren Anteil (unter 20\%) vertreten waren als im Bundestag, keine 2/3-mehrheitsfähige Neigung bestand, derartig weitreichenden Reformwünschen nachzugebon? Die Vorbchalte waren noch wesentlich stärker als gegen die Ergänzungsforderungen zu Art. 3 GG. Dies ist nicht ohne Grund so, denn rechtspolitische Änderungsvorschläge haben paradoxerweise noch am meisten Realisierungsaussichten, wenn sich für die politischen Mehrheitsakteure und deren Bezugskreise letztlich doch nichts oder wcnig verändert. Selbst wenn die volle "Kompensationsklausel « zur Absicherung der Quotierung durchgekommen wäre, könnten sich Bürokratieerfahrene erleichtert mit dem Wissen zurücklehnen, daß ihre Qualifikationsdefinitions- und Entscheidungsmacht im Einzelfall bei Einstellungen und Beförderungen doch sehr vieles von dem relativieren wird, was das Gebot der Frauenbevorzugung zunächst als absolut suggeriert. Mit dem gesetzlichen $Z$ wang, vorrangig Frauen einzustellen, ist auf diese Weise bequem zu leben. Im Fall der Entprivilegierung der Ehe sind die möglichen Folgen dagegen unabsehbar und weitreichend, der Abbau von Transferungerechtigkeit zwischen Kinderhabenden und Kinderlosen ${ }^{3}$ und die Einebnung der sozialpolitischen Statusunterschiede von Ehe und Nicht-Ehe (s. u.) betreffen vor allem jene gutverdienenden Männer mit familienorientierten Ehefrauen, ${ }^{4} \mathrm{zu}$ denen auch sehr viele Abgeordnete und ihre politischen Freunde und Berater zählen, so daß die kommissionsinterne und allgemein-politische Abneigung gegen derart Revolutionärcs durchaus vcrständlich ist.

\section{Ehe und Familie im Grundgesetz}

Im folgenden sollen - mit dem Schwerpunkt auf Art. 6 Abs. x GG - folgende Fragestellungen eingehender behandelt werden: Was haben die (herrschenden) Verfassungsinterpreten der Bundesrepublik - Verfassungsrechtlerinnen gab und gibt es kaum - bisher als Inhalt und Funktion von Art. 6 GG angesehen? Inwieweit prägt

I Kuratorium fur eınen demokratısch verfaßten Bund deutscher Lander: Von Grundgesetz zur deutschen Verfassung. Denkschrift und Verfassunusentwurf. Baden-Baden 1991.

2 So sah es der Verfassungsentwurt des Runden Tisches der DDR in Art. 22 vor: n(1) Die Familie ist durch den Staat zu schutzen und zu fordern. (2) Andere Lebensgemeinschaften, die auf Dauer angelegt sind, haben Anspruch auf Schutz vor Diskrıminierung." Vgl. Arbeitsgruppe "Neue Verfassung der DDR*, Berlin April 1990.

3 Suhr, Dieter: Transferrechtliche Ausbeutung und verfassungsrechtlicher Schutz von Fanilien, Mutcern und Kindern, in: Der Staat, 1990, S. $69 \mathrm{ff}$. Oeter, Ferdinand: Die mißachteten Mutter, in: FamRZ Heft 6/1990, S. 584-588; derselbe: Transferbelastung der Familic, in: FamRZ 1991, 1025 -1028; Borchert, Jurgen: Man muß kein Extremist sein... Famulie im Transferrecht: nur ein sozialpolitisches Problem?, in: FuR Heft 2/92, S. 88-98.

4 Becker, Ruth: Lohnt die Ehe wirklich? Broschure der GRUNEN im Bundestag, Bonn 1990. 
dieses Verständnis auch die Entwicklungsmöglichkeiten des Rechts auf der einfachgesetzlichen Ebene und der gesellschaftlichen Geschlechterverhältnisse? Welche Veränderungen wurden diskutiert, und wie müßte ein Grundrecht der Familie und Zweierbeziehung aussehen, das von religiösen, bevölkerungspolitischen und autoritären Ordnungsvorstellungen befreit ist?

\subsection{Bestandteile des Grundrechts}

Wortlaut und Bedeutung von Art.6 GG machen wie bei kaum einem anderen Grundrecht deutlich, daß der gcsellschaftliche Wandel in den Verhältnissen und Anschauungen schon in gut 40 Jahren vermeintliche Ewigkcitswerte zu problematischen Festschreibungen werden lassen kann. Absatz i stellt die Ehe und die - bislang von der herrschenden Meinung - über sie definierte Familie (s. u.) unter den besonderen Schutz der staatlichen Ordnung. Damit verbunden war früher die Vorstellung, daß allein in der Ehe Sexualität und Fortpflanzung legitim stattfinden dürfen und daß tatsächlich in den allermeisten Ehen auch Kinder geboren und crzogen werden. Die Förderung der Ehe würde also automatisch die Förderung von Familiengründungen und Geburten zur Folge haben. Heute aber gibt es neben den Ehen, die immer brüchiger werden, sehr vielc nichteheliche Lebensgemeinschaften, auch solche mit Kindern. Angesichts einer allgemeinen Pluralisierung in den Formen des Zusammen- oder Alleinlebens's werden nichteheliche Beziehungen und Familien zunehmend akzeptiert, so daß sich die Frage stellt, ob die Privilegierung der Ehe noch zeitgemäß ist und nicht sogar allgemcinen Gcrechtigkeitsvorstellungen von sozialem Ausgleich im gesellschaftlichen "Generationenvertrag" widerspricht.

Art. 6 GG definiert ferner - in bewußter Abgrenzung zum Nationalsozialismus und totalitären Tendenzen in kommunistischen Staaten - die Kindererziehung als »das natürliche Recht der Eltern und die zuvörderst ihnen obliegende Pflicht « (Abs. 2). Über die Betätigung darf der Staat lediglich "wachen«, eingreifen darf er nur im Notfall, d. h. bei Versagen der Erziehungsberechtigten oder anderweitiger Gefahr der Verwahrlosung (Abs. 3). Das Grundrecht hat in dicsen Absätzen einen vorrangig abwehrrechtlichen Charakter, überläßt deshalb aber auch allzuviel an gesellschaftlicher Aufgabenerfüllung allein den Eltern, d. h. auf der Alltagsebene Frauen, die aus diesem Grund zusätzlich beruflich benachteiligt werden. Daß der Staat beiden Elternteilen, d.h. eben auch Müttern, helfen solltc, Erwerbstätigkeit und Familie zu vereinbaren, war zu Beginn der Bundesrepublik gewiß kein Thema, im Gegenteil! Das Leitbild der Hausfrauenehe galt weitgehend unangefochten in Politik, Recht und Gesellschaft, was dem Staat u. a. den Ausbau von offentlicher Kinderbetreuung und von Arbeitsmarkt- und Qualifizierungsförderung für Frauen ersparte und Männer vor weiblicher Konkurrenz um Positionen und Bezahlung abschottetc. Der Gegensatz zur in Art. 3 Abs. 2 GG umfassend garantierten Gleichberechtigung fiel kaum auf. Die Kindererziehung ist im Grundgesetz zudem lediglich als Recht der Eltern akzentuiert, nicht als Anspruch der Kinder auf optimale Entwicklung und Entfaltung; daß Kinder Grundrechtsträger sind, versteht sich zwar von selbst, geht aber im Grundrechtstext des Art. 6 GG bisher völlig unter.

s Es haben sich nicht unbeding die familiaren Formen des Zusammenlebens vermethrt; domınanı bleıbt weıter die Eltern-Kınd-Familie, starken Zugewinn haben aber die Allenerziehenden bzw. die Einelternteil-Familie (eventuell mit neuem Partner) zu verzeichnen. Vor allem jedoch ist die Zahl derjenigen gestiegen, die in anderen Lebensformen wie Ein-Personen-Haushalten oder nichtehelıchen Lebensgemeinscbaften ohne Kinder leben. Vgl. Nave-Herz, Rosemarie: Die Pluralitat von Familienformen: Ideologie oder Realitát. In: FuR Heft $4 / 1992$, S, 186-191. 
Die von Art. 6 GG vorgesehene "Normalform« des Zusammenlebens ist die Ehe und die eheliche Kleinfamilie, dennoch soll - nach dem Willen der Verfassungseltern auch jede Mutter den „Anspruch auf Schutz und Fürsorge der Gemeinschaft « haben (Abs. 4). Dieses Grundrecht bleibt nnotorisch unterschätzt ${ }^{6}$. Man meint, aus ihm folge - ähnlich wie aus der Teilhabedimension des "besonderen Schutzes* der Familie (s. u.) - wenig Konkretes, was über den engeren Mutterschutz und die Bewahrung der Gebärfähigkeit hinausgeht, obwohl es doch eigentlich naheliegen müßte, in diesem Absatz einen verstärkten sozialen Schutz- und Förderungsanspruch für alleinerziehende Mütter und Väter zu sehen. Dieser wäre jedoch entbehrlich, wenn er bereits unter den Schutz- und Förderungsanspruch der "Familie" in Abs. 1, zu der heute unbestrittenermaßen auch der alleinerziehende Elternteil mit Kind - sei es nichtehelich oder ehelich - gehört, subsumiert wird. Tatsächlich erkennt auch die herrschende Meinung in Abs. 4 ein subjektives öffentliches Recht und sogar eine Institutionsgarantie, ${ }^{7}$ aber beschränkt auf "Mütter «, so daß es unklar bleibt, was die Garantie umfassen soll, wenn nicht eine biologische Tatsache, die wohl kaum eine Rechtsinstitution darstellen kann $;^{8}$ es fehlt auch für das subjektive Recht gerade bei der Interpretation von Teilhabeaspekten an wirksamen Kriterien, die einen Kern von Leistungsansprüchen gegen den Staat, also von sozialen Grundrechten definieren.

Den Abschluß des Grundrechts bildet ein Verfassungsauftrag, der in der Weimarer Zeit nicht erfüllt worden war und deshalb schon 1949 als historisch überfällig erneuert wurde: die Gleichstellung der "unehelichen " mit den ehelichen Kindern in den "leiblichen « und "seelischen « Entwicklungsbedingungen. Dieser Auftrag wurde erst 1969/70 formal erfüllt, führte aber substantiell nur zu einer halbherzigen Angleichung in der Rechtsstellung. Mehr als die nichtehelichen Kinder selbst werden heute noch ihre Mütter diskriminiert (z. B. durch die automatische Amtspflegschaft des Jugendamtes für nichteheliche Kinder), in mancher Hinsicht auch ihre Väter, wenn diese die soziale Vaterschaft ernst nehmen; all dies wirkt sich selbstverständlich auf die Entwicklungsbedingungen der betroffenen Kinder aus. Ehelichkeit oder Nichtehelichkeit als solche ist heute dennoch nicht mehr unbedingt das für die Lebenschancen von Kindern entscheidende Differenzierungs- bzw. Diskriminierungskriterium. Soll ein sozialstaatliches Angleichungsgebot oder ein subjektiver Anspruch auf Nicht-Diskriminierung verfassungsrechtlich verankert werden, so müßte sich daher die Formulierung auf "allc « Kinder beziehen. ${ }^{?}$

6 Eva Maria von Münch, Thesen zur Stellungnahme fur dic offentliche Anhorung vor der Gemeinsamen Verfassungskommission am 10. 12.1992, Manuskript, S. 4 .

7 Maunz/Durig/l lerzog, Kommentar zum Grundgesetz, Munchen Stand: September 1980, Anm. I g.d. zu Art. 6.

8 Das Grundrecht kann nach einem modernen Verstandnis von sozialer Elternschaft und grundsatzlicher Eignung auch des Vaters fur die Kindererzichung nicht bedeuten, daß die Mutter im Verhaltnis zum Vater auf Grund der biologischen Tarsachen eine rechtlich abgesicherte engere soziale Beziehung zum Kind beanspruchen kann. Frau/man konnte die institutionelle Interpretation als eine Reaktion auf die nationalsozialistische: Gebarpolitik verstehen, weil es dor bestimmten Frauen aus rassıschen oder eugenischen Grunden untersage wurde, Kinder zu bekommen. Daruber hinaus ist es seit der forcierten Entwicklung der Reproduktionsmedizin und von Forschungsarbeiten an einer künstlichen Gebarmutter vorstellbar, daß auch die biologische ' Fatsache, daß alle Menschen durch Mutterschaft zur Welt kommen, rechelich in Frage gestellt werden konnec.

9 So auch dic Forderungen von Michael Coester, Barbara Degen, Ute Gurhard, Eva Maria von Minch in der offentlichen Anhorung vor der Gemeinsamen Verfassungskommission von Bundestag und Bundesrat am 10. 12. 1992, vgl. Manuskripte (Ms.). Inzwischen auch als Stenographischer Bericht der 6. Anhörung hekthographiers erhalelich. 
Das Grundrecht des Art. 6 GG ist einerseits ein Abwehrrecht gegen staatliche Eingriffe in die private Lebensgestaltung, andererseits aber nach herrschender Mcinung auch eine "Institutionsgarantie«, d. h. ein Verbot der Aushöhlung der Substanz von Ehe, Familie und Elternrecht durch einfache Geserze. Dies gilt insbesondere für die Begriffe von "Ehe" und "Familie «. Daneben wird in Abs. I noch eine "wertentscheidende Grundsatznorm « gesehen, ${ }^{10}$ wobei diese Deutung im wcsentlichen als dekkungsgleich mit der institutionellen Auffassung angesehen wird.'

Im Hinblick auf das in Art. 6 Abs. I GG enthaltene Abwehrrecht bedeutet der Schutz der Ehe, daß die BürgerInnen die Freiheit haben müssen, wihren religiösen und weltanschaulichen Verpflichtungen mit allen Konsequenzen nachzuleben ". ${ }^{22}$ Darunter fällt nach herrschender Auffassung auch die Entscheidung über die eheliche Arbeitsteilung zwischen Beruf und Familie. Laut Bundesverfassungsgericht wäre eine Bestimmung, die die Ehefrau »ins Haus zurückholt «, verfassungswidrig, ${ }^{13}$ aber - so läßt sich schließen - auch eine Rechtsgestaltung wie z. B. in der DDR, die beide Ehegatten faktisch zur Erwerbstätigkeit zwang. Nachdem aber mit dem I. EheRG 1976/77 die Hausfrauenche als Leitbild abgeschafft wurde und für die Zeit nach der Scheidung - z. T. schon während der Trennung - eine grundsätzliche Erwerbspflicht für beide Ehegatten, also auch für die zuvor nicht erwerbstätige Frau festgeschrieben wurde, hat - jedenfalls für jüngere kinderlose Ehefrauen - die Wahlfreihcit, zu Hause zu bleiben und nur dem Ehemann den Haushalt zu führen, gesellschaftlich und rechtspolitisch erheblich an Legitimität eingebüßt. ${ }^{14}$ Gleichwohl bleibt das Problem für Frauen mit Kindern oder ohne, daß sie dennoch und oft kontrafaktisch, geradezu mit ciner Art Double-Bind-Logik, auf dem Arbeitsmarkt und im Sozialsystem auf die Rolle der abhängigen Nicht-Erwerbstätigen oder $\mathrm{Zu}$ verdienerin verwiesen werden und ihnen so eine eigenständige Existenzsicherung, insbesondere für das Alter, massiv erschwert wird. Steuer- und Sozialrecht - im Hinblick auf seine scheinbar perfekten Absicherungsverheißungen sogar das Scheidungsunterhaltsrecht - fördern immer noch die Konstellation der Hausfrauenehe und -familie und lassen andere Formen der Arbeitsteilung und der Familie, wie z. B. alleinerziehende Frauen mit Kindern oder unverheiratetc Paare mit Kindern, ohne ausrcichende Unterstützung. Der eherechtlich proklamierte Gestaltungspluralismus in Sachen Arbeitsteilung existiert also nicht wirklich.

Aus der institutionellen Interpretation folgt für die bislang herrschendc Rechtsauffassung, daß die Ehe keineswegs als Vertrag zu bctrachten ist, d. h. als rein private Angelegenheit der Beteiligten, vielmehr soll es sich um eine »überindividuelle Privatrechtsinstitution " handeln, " also um eine vorgegebenc Konstruktion, die von den Beteiligten nicht beliebig abänderbar ist. Ehe und Familie knüpfen nach herrschender Auffassung an "vorstaatliche, naturgegebene Ordnungen « an. ${ }^{16}$ Es wird daher vielfach angenommen, die Familie sci die "Keimzelle der Gesellschaft $\alpha$ oder sogar

Io Maunz/Durig/Herzog, (Fn. 7), Anm. I s.a) zu Ar. 6.

If Friaut, Karl Heinrich: Verfassungsgarantie und sozialer Wandel, in: NJW Heft 42/1986, S. $2595-2602$, 2600.

12 BVerfGE $10,59 \mathrm{ff} ., 83$.

13 BVerfGE $21,329 \mathrm{ff} ., 353$.

I4 Vgl. die heftige offentliche Diskussion in Hessen um entsprechende Äußerungen der ehemaligen hessischen Ministerin fur Frauen, Arbeit und Sozialordnung, Heide Pfarr, daß allein das Hausfrauendasein keine rechtliche und steuerlichc Forderung mehr beanspruchen konnc. Mit ahnlicher Tendenz argumencierte sction 1968 Thilo Ramm: Gleichberechugung und Hausfrauenche, in: IZ 1968, S. 41-46, 94-94

is Palande-Diederichsen, Kornmentar zum BGB, 37. Aufl. Munchen 1978 , Einfl. 1 vor $\$ 1353 \mathrm{~m}$. w. N.

16 Maunz/Durig/Herzog, (Fn. 7), Anm. II t. zu Art. 6. 
des Staates und "Bauglied des politischen Lebens «. ${ }^{17}$ Dahinter steht offenbar ein relikthaftes stratifiziertes Gesellschafts- und Staatsverständnis, ${ }^{18}$ wonach eine (hierarchische) Gesellschaftsstruktur bzw. ein entsprechender Staatsaufbau die vergröBernde Projektion der Familienstruktur sei oder - weitergehend - sogar mit dem familiären Herkunftsmilieu schon der Platz in Gesellschaft und Politik maßgeblich determiniert werde. Zumindest offenbart sich bei den "Keimzellentheoretikern « ein ordnungspolitisches Bedürfnis, über die rechtliche Formierung des Zusammenlebens die Gesellschaft zu strukturieren. "' Der Widerspruch, daß Ehe und Familic zwar als anthropologisch und historisch vorgegeben gelten, die Begriffe und ihre prägnante Ausformung aber ausschließlich dem positiven Recht entnommen (und dabei auch relativ jung) sind, wird zwar in der Kommentarliteratur zugestanden, ${ }^{z 0}$ führt jedoch nicht zu einer Infragestellung der aus dieser institutionellen Sicht abgeleiteten Rechts- und Interpretationsfolgen. Diese bestehen vor allem in der Ausgrenzung von solchen Konstellationen, die nicht den Schutz von Ehe und Familie beanspruchen dürfen.

Das Bundesverfassungsgericht faßt unter die vom Grundgesetz geschützte Ehe "die lcbenslange Verbindung eines Manncs mit einer Frau zur Lebensgemcinschaft "." Gleichgeschlechtliche Verbindungen sind somit ausgeschlossen. Darüber hinaus muß die Ehe formell geschlossen werden. ${ }^{23}$ Die "nichteheliche Lebensgemeinschaft " steht damit nicht unter dem Schutz des Art. 6 Abs. I GG, wobei von Verfassungsinterpreten oft sofort aus der Unterlassung der förmlichen Eheschließung auf eine Ablehnung der Ehe als Lebensform geschlossen und deshalb auch ausdrücklich nicht von weheähnlichen Gemeinschaften " gesprochen wird. ${ }^{23}$ Dic Ablehnung der Ehe als Rechtsinstitution wird wiederum oft auf bewußt fehlenden Bindungswillen »des Paares « zurückgeführt, wobei häufig nicht bedacht wird, daß eine Eheschließung auch dann nicht zustande kommt, wenn nur bei einem Teil der Wille dazu fehlt. Empirische Ergcbnissc über ganz unterschiedliche Gründe und Motive, eine Ehe obwohl möglich - nicht oder noch nicht einzugehen, ändern offenbar nichts an der Überzeugung vieler Verfassungsinterpreten, daß man es hier mit überzeugten Gegnern der rechtlich abgesegneten "Keimzelle» zu tun hat, dic für ihre Gegnerschaft nicht noch mit den Vorteilen der Ehe bclohnt werden sollen. ${ }^{24}$

Auch der Begriff »Familie", der weit mehr als der der Ehe einer lebensweltlich ausgeweiteten Interpretation zugänglich ist, wird von der Rechtsprechung und Literatur

17 Rosenau, Heinz: Die Familie in privatrechtlicher und steuerrechtlicher Hinsicht. Hildesheim 197r, S. 2 .

18 Vgl. Luhmann, Niklas: Frauen, Manner und George Spencer Brown, in: Zettschrift fur Soziologie, Heft 1/1988, S. 47-91, 59f.; derselbe: Die gesellschaftliche Differenzierung und das Individuum, in: Olk, Thomas/Otto, Hans-Uwe (Hg.): Soziale Dienste ım Wandel i. Helfen im Sozialstaat. Neuwied/Darmstadt 1987 , S. $121-137$.

19 Vgl. Campenhausen, Axel Freiherr von: Verfassungsgarantie und sozualer Wande]-Das Beispiel von Ehe und Familie. In: Veroffentlichungen der Vereinigung der Deutschen Staatsrechtslehrer, Heft 45 , Berlin, New York 1987, S. 7 ff., zugleich Stellungnahme in der Anhorung vor der Gemeinsamen Verfassungskommıssion von Bundestag und Bundesrat am 10. 12. 1992. Ahnlich in der $A$ rgumenration: Loschelder, Wolfgang: Staatlicher Schutz fur Ehe und Familie. Der Auftrag der Verfassung und die Krise der Institution, in: FamRZ Heft $4 / 1988$, S. $333^{f f}$

20 Maunz/Durig/Herzog, (Fn. 7), Anm. II i zu Art. 6.

21 BVerfGE 10, 59 ff., 66; vgl. auch Maunz/Durig/Herzog,, (Fn. 7), Anm. II 2 zu Art. 6.

22 Ebenda.

23 Ehenda. Anders BVerfG vom 17.11.1992, Az. 1 BvL 8/87 sowic BSG vom 24.3.1988, NJW Heft $34 / 1988$, S. 2128 ff. Hier wird von der -eheahnlıchen Lebensgemeinschaft gesprochen. Fur sie setze das BVerfG gegensettige soziale Einstandspflichten der PartnerInnen voraus, die sozıaltypisch seien und die von den Betroffenen im Großen und Ganzen auch subjektiv in thr Bewußtsein aufgenommen wurden. Dies ist aber cine neuere Interpretationsrichtung, s. u.

24 Vgl. Campenhausen, A. Freiherr von, (Fn. 19), S. 17. 
mehrheitlich noch immer eng und ausgrenzend gefaßt. Familie ist demnach nicht schon die Ehe ohne Kinder, aber auch nicht die Großfamilie mit drei und mehr Generationen und/oder weitläufigen Verwandten. Unter Familie fällt vielmehr nur die Klein- oder Kernfamilie von Eltern und Kindern, obgleich diese nicht notwendig zusammenleben müssen. ${ }^{25}$ Die nichteheliche Lebensgemeinschaft ist aber nach bisher herrschender Meinung auch dann keine Fanilie im Sinne des Grundgesetzes, wenn gemeinsame Kinder vorhanden sind. Selbst mancher Kommentator findet es "seltsam«, daß zwar sowohl die Mutter mit ihrem nichtehelichen Kind als auch der Vater mit dicsem einc Familie bilden, es sich aber um zwei Familien handeln sol], nicht um eine einheitliche. ${ }^{26}$ Es gehört nicht vicl Spürsinn dazu, für diese konstruktive Akrobatik den institutionellen Ehcbegriff und die hinter ihm stehendc ordnungspolitische Gestaltungsabsicht "staatstragender " Kräfte auszumachen.

So schützt Art. 6 Abs. I GG zwar auf den ersten Blick das Teilhaberecht der Verheiratcten an schützenden und fördernden Maßnahmen, aber hicr stehen wiedcrum dic negativen Pflichten des Staates im Vordergrund, d. h. die Unterlassung von Schädigungen oder Beeinträchtigungen. Eheerhaltende Elemente müssen demnach für den Gesetzgeber stets maßgeblich sein, wenn auch der Gestaltungsspielraum rccht groß ist. ${ }^{27}$ Aus diesem Gebot wird sogleich das Verbot für den Staat abgeleitet, nichtehelichc Lebensgemeinschaften gleich-, geschweige denn besser zu stellen. ${ }^{28} \mathrm{Dic} \mathrm{Be}-$ nachteiligung anderer $Z$ weierbeziehungen folgt so aus der vorgeschriebenen Privilegierung. Dem »besonderen "Schutz durch die staatliche Ordnung wird in Literatur und Rechtsprechung keine Höherwertigkeit gegenüber einem Schut $z$ überhaupt eingeräumt; ${ }^{29}$ vielmehr differenziert man(n) zwischen Schutz und Nicht-Schutz und spricht den nichtehelichen Formen der Zweisamkeit somit den spezifischen Schutz des Art. 6 Abs. I ab. Ansonsten ist es bisher in der positiven Leistungs- und Teilhabedimension des Grundrechts lediglich bei eincm vage gchaltenen Differenzierungsgebot zugunsten der wirtschaftlichen Entlastung von kinderreichen Familien geblieben. ${ }^{3 \circ}$ Die Gestaltungsfreiheit des Gesetzgebers bei der Familienförderung ist demnach denkbar groß; die Grenze wird erst da gezogen, wo sich der Gesetzgeber der Aufgabe der Ehe- und Familienförderung überhaupt entzichen würde. ${ }^{\prime \prime}$

Auf diese Weise werden ebegeneigte BürgerInncn "für ein Linsengericht « mit crheblichen ordnungs- und sozialpolitischen Erwartungen konfrontiert; nichtehelichc GefährtInnen müssen sogar fast ausschließlich Pflichten erfüllen. Funktional betrachtet ist es also weniger der Staat, der Ehe und Familie als Lebenssachverhalte schüt $z t$, es ist vielmehr die rechtliche Institution Ehe, dic die Familie und vor allem das gesellschaftliche Verhältnis zwischen den Geschlechtern strukturiert und für die staatliche Ordnung nutzbar und steuerbar macht. Die Rechtskonstruktion schürzt nicht einen lebensweltlich vorstrukturierten Bereich vor staatlichen Eingriffen, sondcrn kanalisiert ihn von vornherein durch staatliche Regelungen. Der sozialpolitische Transformationsriemen zwischen diesem institutionellen Verfassungsverständnis und der einzelnen Ehc bzw. Familic ist das Subsidiaritätsprinzip, das die vorrangige Verpflichtung von Ehegatren und Vervvandten vor Sozialleistungsträgern festlegt, d.h. den Staat im Bedarfsfall oft von der Leistungspflicht freistellt. Dcr sozialpolitische Spareffekt wird vor allem am Beispiel der Sozialhilfe oder Arbeits-

25 Maunz/Durig/Herzog, (Fn. 7), Anm. II 3 zu Arr. 6.

26 Ebenda.

27 Ebenda, Anm. II 4 zu Arr. 6

28 Ebenda, Anm. II 4 b) zu Art. 6 .

29 Ebenda, Anm. I sa) zu Art. 6.

30 I:benda, Anm. II 4.b) zu Art. 6 .

3 I Ebenda. 
losenhilfe sichtbar. Daß hiermit auch die Asymmetrie des Geschlechterverhältnisses verfestigt wird, hat das Bundesverfassungsgericht in jüngster Zeit festgestellt. ${ }^{32}$ Die bisherigen Anrechnungsregeln des Einkommens von Ehegatten und nichtehelichen Lebensgefährten bei der Arbeitslosenhilfe und insbesondere die niedrigc Freibetragshöhc üben, so der Spruch aus Karlsruhe, cinen mittelbaren ökonomischen Zwang zur Hausfrauenehe aus und wirken damit geschlechtsspezifisch benachteiligend. Während das Gericht die spezifischen Anwendungsregeln des Subsidiaritätsprinzips im AFG vcrwarf, ließ es den Grundsatz als solchen und seine Ausdehnung auf nichteheliche bzw. eheähnliche Beziehungen, die im AFG erst seit 1986 vcrankert ist, aber auch zuvor praktiziert wurde, im wesentlichen unangetastet. Kennzeichnend für die Bedürftigkeitsprüfung ist und bleibt im Grundsatz, daß der Begriff nicht auf die einzelne Person bezogen wird, sondern auf die Gemeinschaft von zusammenlebenden Ehegatten, nichtehelichen Lebensgefährten oder - eingeschränkter - von Eltern und erwachsenen Kindern. Daß die somit nicht mehr Bedürftigen in die persönliche Abhängigkeit von ihren (Ex-)Partnern oder Eltern gedrängt werden und häufig berufliche und qualifikatorische Entfaltungsmöglichkeiten nicht wahrnehmen können, wurde bislang als zwangsläufige Folge des an sich Privatheit und Mitmenschlichkeit bewahrenden und fördernden Subsidiaritätsprinzips in Kauf genommen oder sogar als gerecht befunden. Erst das erwähnte jüngste Urteil des Bundesverfassungsgerichts achtet zumindest stärker darauf, daß die einzelnen PartnerInnen nicht mit allzu pauschalen und unrealistischen Einstandserwartungen überzogen werden, die ihre individuelle Handlungsfreiheit unverhältnismäßig einschränken.

Der von dem privaten Einstandsprinzip traditionell und idealtypisch Verpflichtete ist der männliche Allein- oder Hauptverdiener in eincr bürgerlichen mittel- bis oberschichtigen Ehe bzw. der Familienvater. Damit er seiner Pflicht überhaupt einigermaßen nachkommen kann, was ökonomisch immer schwieriger wird, und damit er sich für das Häusliche und Familiäre eine (allenfalls in zweiter Linie auch erwerbstätige) Hausfrau und Kindererzieherin leisten kann, die auf diesc Weise einen Gegenwert für die statusrechtlich erzwungene - mehr oder weniger tatsächliche Versorgung schafft oder zu schaffen angehalten wird, gibt es vor allem das "Ehegattensplitting im Steuerrecht. Dieses wirkt mittlerweilc dysfunktional und wirft die Frage nach seiner Verfassungsmäßigkeit auf. Es begünstigt nämlich in sozial- und geschlechterpolitisch anachronistischer Weise die kinderlose "Hausfrauenehe", verhilft also - auch verheirateten - Menschen mit Kindern nicht zu einem wirklichen steuerlichen Ausgleich ihrer - auch für die Gesellschaft getragenen - materiellen Belastungen. 33 Bekanntlich wurde das Splittingsystem in den fünfziger Jahren als Reaktion auf eine Verfassungsgerichtsentscheidung geschaffen, welche die zuvor geltende, extrem frauenerwerbstätigkeitsfeindliche progressive Besteuerung des addierten Einkommens beider Ehegatten für gleichberechtigungswidrig crklärt hatte. ${ }^{34}$ Aus der konservativen Frauen- und Familienperspektivc dieser Zeit gesehen ist es folgerichtig, daß zwar die offene Benachteiligung von Frauenerwerbstätigkeit beseitigt wurde, derselbe Effekt aber nun auf mildere Weise, nämlich durch einen steuerlichen Anreiz zur Hausfrauenehe erzielt wurde. Es erscheint jedoch unverständlich, warum nach mehreren Jahrzehnten, die bedeutende familien - und gleichberechtigungspolitische Reformen mit sich gebracht und das Leitbild der Hausfrauenehe abgeschafft habcn, das Ehegattensplitting von politischen Mehrheiten immer

32 BVerfG vom 17.11. 1992, Az., B BL 8/87, Ms. S. 37 .

33 Becker, Ruth, (Fn. 4), S. 12, 32 If.

34 BVerfGE 6, ssff. 
noch als berechtigt und angemessen angesehen wird. Zum einen Teil bietet sich zur Erklärung hier die rechtspolitische Verhinderungsdynamik hinsichtlich solcher Änderungen an, die die politischen Akteure und ihr Umfeld gravierend selbst betreffen (s. o.). Zum anderen Teil mag das hartnäckige Festhalten am Ehegattensplitting damit zu erklären scin, daß die indirekte steuerliche Absicherung der Hausfrau und insbesondere der Mutter kleiner Kinder den Staat immer noch wesentlich billiger kommt als wirksame arbeitsmarkt- und sozialpolitische Förderungsmaßnahmen für Frauen und Mütter, wozu in erster Linie auch der Ausbau der öffentlichen Kinderbetreuung gehören würde. Die Kinderbetreuungssituation hat sich zwar-auch im Westen - seit den fünfziger Jahren erheblich verbessert, ist dort längst aber nicht bedarfsgerecht und rangiert im europäischen Vergleich ziemlich weit hinten."

Zusammenfassend läßt sich feststellen, daß nicht der lihe- und Familienschutz als grundlegendes Abwehr- und Freiheitsrecht im Vordergrund der Verfassungsinterpretation steht, auch nicht die sozialstaatliche Förderung der Familie, sondern die Sicherung und weitere ordnungspolitische Nutzbarkeit der Ehe als Rechtskonstruktion und - auf ihr aufbauend - der ehelichen Familic. Daß der Staat sich gleichwohl mit Eingriffen in die "Intimsphärc z zurückhält, ist vielen, vor allem historischen Gründen und Motiven geschuldet, so zum Teil auch einem patriarchalischen Verständnis von Ehe und Familie, ${ }^{36}$ ist zum größeren Teil aber wiederum als Gegenleistung bzw. -unterlassung gegenüber den BürgerInnen für die Akzeptanı der Institution zu verstehen, mit der ordnungs- und sozialpolitische Ziele und Strategien verfolgt werden. Ursprünglich sicherten Ehe und Familie als Institutionen die patriarchale Ordnung37 in sehr viel direkterer Weise. Als Relikte sind auch heute noch z. B. die Nicht-Strafbarkeit der ehelichen Vergewaltigung oder das Bestimmungsrecht des Vaters, wer zur Familie gehört (Nichtanfechtbarkeit der Ehelichkeit eines Kindes durch die Mutter) vorzufinden. Dennoch ist die Konstitution von Zweierbeziehungen und Familie in Deutschland heute unter der Gcltung des Gleichberechtigungsgrundsatzes und nach der wesentlichen Reformierung des Ehe- und Familienrechts eher indirekt patriarchalisch beeinflußt, nämlich durch den Zwang der Umstände und scheinbare Sachgesetzlichkeiten des Arbeitsmarkts und des Sozialsystems. Daß es strukturell auch anders geht, zeigt sich im Vergleich mit skandinavischen Sozialstaatssystemen, die nicht vorrangig unterschiedlichen Status absichern, sondern eher universalistische und am Individuum orientierte Zugangskriterien festlegen. ${ }^{38}$

\subsection{Die Vorteile der Nicht-Ehe}

In der sozialen Realität hält dies alles jcdoch immer weniger Menschen ab, ungeachtet der Nachteile unverhciratet zusammenzuleben. Schwule und Lesben haben diesbezüglich ohnehin bisher keine. Wahl. Angesichts der Anonymität im Verkehr mit Ämtern bietet nicht-verheiratctes Zusammenleben manchmal doch noch den

3s Vgl. Oelschlager, Angelıka/Schunter-Kleemann, Susanne: Frauen als Erwerbsarbeiterinnen - Frauen als Familienarbeiterınnen - Frauen, Eltern, Famılien als Bezıeher sozialer Leıstungen, in: Schunter-Kleemann, S. (Hg.): Herrenhaus Europa, Berlin 1992, S. 329 ff. Positionen 37-40 im Landervergleich.

36 Vgl. Pateman, Carole: The Sexual Contract. Stanford 1988; deutscher Kurzauszug: Der Geschlechtervertrag, in: Narr, Wolf-Dieter/Vack, Klaus (Hg.): Verfassung. Oder: Wie konnen wir in Zukunft leben? Sensbachtal 1991, S. 112-123. Olsen, Francis E.: The Myth of State Intervention in the Family, in: University of Michigan Journal of Law, Vol. 18, Number 4, 1985, S. $835-864$.

37 So auch Monıka Frommel mit Verweis auf dic liberal patriarchale Tradition (pragnant formuliert bei Immanuel Kant), vgl. Eheliches Sonderrecht oder privatautonome Bezichungen?, in: Zeitschrift fur Sozialforschung. Heft $3 / 1991$, S. $181-191,188 \mathrm{f}$.

38 Vgl. I.angan, Mary/Ostner, Ilona, 1991: Geschlechterpolitsk im Wohliahrtsstaaz: Aspekte im internationalen Vergleich, in: Kritische Justiz, Heft 3, S. 302-317. 
Vorteil der leichteren Verschleierungsmöglichkeit, teilweise gibr es aber durchaus legale Vorteile oder zumindest keine Nachteile, weil z. B. die steuerliche Privilegierung eben nicht die Ehe schlechthin honoriert, sondern nur diejenige mit einer extrem asymmetrischen Einkommensaufbringung. ${ }^{39}$ Angesichts der hohen Kosten von Scheidungen, der patriarchalen symbolischen oder tatsächlichen Herabsetzungen von Frauen durch manche Rechrsfolgen der Ehe wie z. B. die Ausgrenzung aus dem Straftatbestand der Vcrgewaltigung, angesichts der auf der anderen Seite nachlassenden sozialen Kontrolle über Sexualität, angesichts besserer und besser zugänglicher Verhütungsmittel und größer gewordener gesellschaftlicher Toleranz und Akzeptanz gegenüber unterschiedlichen Lebens- und Zusammenlebensstilen ist die nichteheliche Gemeinschaft schon fast die "näherliegende " Form, zumindest wenn es um das Ausprobieren des Zusammenlebens geht. Und gerade viele frauenbewegte Frauen, die bezüglich des Sorgerechts für ihre potentiellen Kinder kein Risiko eingehen wollen, heiraten selbst dann nicht, wenn ein Kind »unterwegs « ist, zumal sie z. T. in sozial- und steuerrechtlicher Hinsicht, bei der Vergabe von Sozialwohnungen und neuerdings im Hinblick auf Krankenpflegetage ${ }^{40}$ durchaus Vorteile aus dem Status der Alleinerziehenden ziehen können. Nicht-verheiratete Frauen mit Kind werden - günstigerweise - immer noch in einigen, wenn auch nicht allen rechtlichen Hinsichten selbst dann für alleinerziehend bzw. alleinstehend erklärt, wenn sie mit dem Vater des Kindes zusammenleben. ${ }^{4}$ In der DDR war dies noch ausgeprägter der Fall. ${ }^{42}$ Die Einstufung erklärt sich u. a. als Konsequenz aus dem negativen Bild des Vaters eines nichtehelichen Kindes, der traditionell als verantwortungsloser Erzeuger und bindungsunwilliger Mann angesehen wurde bzw. wird, dem wesentliche Rechte gegenüber seinem Kind vorenthalten werden, damit er die Vorteile der ehelichen Bindung schätzen lernen möge und damit auch für Männer weiterhin ein Anreiz zur Heirat existieren möge..$^{43}$ Besonders um die öffentliche Manifestation derartiger Rechte, z. B. als Elternsprecher in der Schule des Kindes, ringen die Gerichte immer noch mit gegenläufigen Ergebnissen.44

Von verschiedenen sozial wissenschaftlichen Untersuchungen wird heute immer wieder hervorgehoben, daß die Arbeitsteilung in nichtehelichen Gemeinschaften cgalitärer sei als in ehelichen, daß nicht-vcrheiratet zusammenlebende Frauen besser gebildet, beruflich qualifizierter, häufiger erwerbstätig, berufsorientierter und besser bezahlt seien.4s Auch fördere es die eigene Selbstbestimmung und die gesellschaftliche Durchsetzungsfähigkeit, wenn frau schon in der eigenen Partnerschaft alles durch Verträge, Vollmachten, Testament u.ä. zu regeln lernt, was ihr wichtig und teuer ist, aber meist gesetzlich vorenthalten bleibt. So gesehen müßte geradezu das

39 Becker, R., (Fn. 4), S. 25 f.

40 Seit I. I. 1992 konnen alleinerzichende Elterneile (oder als solche geltende) 20 Krankenpflegctage pro Kind und Jahr beanspruchen, bei mehreren Kindern allerdings nicht mehr als so pro Jahr, verheiratete Versicherze dagegen pro Elternteil, Kind und Jahr nur 1o, höchstens 25 ( $\$ 45$ SGB V).

41 Z. B. hinsichtlich des Haushaltsfreibetrags, der Kinderbetreuungskosten im Steuerrecht, bei der Berechnung des Kindergeldes, Genaueres vgl. Becker, R., (Fn. 4), S. 32 f. Des weiteren im BAföG; auch zähls fur die Berechnung des Erziehungsgeldes bei nicht-verheirateten Müttern nur das eigene Einkommen, so daß sie wahrend des ganzen Erziehungssurlaubs« dic 600 DM erhalten, vgl. \$6 Abs. I und 3 BErzGG. Frau/man darf gespannt sein, wie das "Alleincrziehen « im neuen \45 SGB V von Arbeitgebern und Krankenkassen bei nicht-verheirateten zusammenlebenden Eltern in Zukunft interpretier wird.

42 Berghahn, S./Fritzsche, A.: Frauenrecht in Ost und West (Deutschland), Bilanz und Ausblick, Berlin 1991, S. $176 \mathrm{f}$.

43 Noch 1981 hatte das BVerfG so argumentiert, vgl. BVerfG vom 24-3.1991, FamRZ Heft s/1991, S. $429 \mathrm{ff}$.

44 Zugunsten des Vaters VG Berlin vom 2.3.1989, NJW 1989, S. 2413-24I5; ablehnend dagegen VGH Baden-Würtcemberg, Az. $9 \mathrm{~S}_{2345} / 90$, vgl. Süddcutsche Zeitung vom 10./11. 10. 1992.

45 Vgl. Stiegler, Barbara: Zur Aufhebung des Eheprivilegs im Grundgeserz. Publikation der Friedrich Ebert Suftung, Bonn 1992, S. $20 \mathrm{ff} .$, m.w.N. 
Nicht-Heiraten als Mittel zur Emanzipation von Frauen propagiert werden. All dies wird naheliegenderweise auch von seiten des Bundesjustizministeriums als Argument benutzt, um mangelnden Handlungsbedarf für die "Verrechtlichung" nichtehelicher Beziehungen hinsichtlich der PartnerInnenebene zu begründen, wobei Verrechtlichung und Gleichstellung der Nicht-Ehe mit der Ehe synonym behandelt werden. ${ }^{+6}$

Beide Argumente greifen zu kurz. Frauen, die in nichtehelichen Beziehungen lcben, stellen gegenüber solchen, die in Ehen leben, eine egalitärer orientierte Vergleichsgruppe dar, weil gebildete, beruflich ambitionierte und selbstbewußte Frauen häufiger ökonomisch selbstständig sind und Vorbehalte gegen symbolische und tatsächliche Abhängigkeitsverhältnisse haben, so daß sie tendenziell weder auf die Versorgung durch einen Ehemann angewiesen sind, noch diese wünschen. Es wird also eher umgekehrt "ein Schuh daraus", indem die »emanzipierten « Frauen auf eine egalitärc Beziehungsstruktur mehr Wert legen und sich dies auch persönlich leisten können. Würde die Nicht-Ehe bei gleichbleibender Gesamtrechtslage auch für die anderen traditioneller eingestellten und/oder ökonomisch oder sonstwie marginalisierten Frauen zur Normalform des Zusammenlebens, so würde ihnen diese Lebensform keine Verbesserung bringen, allenfalls eine noch stärkere Doppelbelastung und andere Zumutungen. Allerdings gilt auch für die Ehe, insbesondere für das Scheidungsrecht, daß sie bzw. es dic zugeschriebenc Versorgungsfunktion nicht mehr leisten kann, jedenfalls nicht leistet. ${ }^{77}$ In der Tat scheinen sich deshalb konservative mit frauenpolitischen Befürchtungen zu treffen, wenn für Gegenwart und Zukunfr ein zunehmendes männliches Interesse an nichtehelichen Beziehungen ohne gesetzliche Verpflichtungen auf der Partnerschaftsebene, aber mit verbesserten Väterrechten konstatiert bzw. vorausgesagt wird. So gesehen verschleiert die liberale Position des Bundesjustizministeriums nur, daß sich die prakrischen Probleme von $Z$ weierbeziehungen und Familien auf Dauer weder dadurch befriedigend lösen lassen, daß den Beteiligten - wie gehabt - die Wahl zwischen Ehe und Nicht-Ehe bleibr, noch dadurch, daß die Nicht-Ehe der Ehe angeglichen wird, was in der Nachteilsdimension ohnehin schon der Fall ist. Vielmehr müßte es darum gehen, ein differenzierteres Schutz- und Förderungsangebot des Staates zu schaffen, das ziclgerichret die funktionalen Bedürfnislagen der - begrifflich weiter als bisher gefaßten - Familie und - in geringerem $\mathrm{Maße}$ - auch der Zweierbezichungen abdeckr, und dieses im Grundsatz, d.h. verfassungsrechtlich, festzuschreiben. Im Rahmen einfacher Gesetze sollcen weitergehend die abhängigmachenden ordnungs- und sozialpolitischen Funktionserwartungen an die Beziehungs- oder Familienmitglieder soweit wie möglich reduziert, Kosten dafür angemessen sozialisiert und Raum für eine die einzelne Existenz und die persönliche Selbstbestimmung besser sichernde Binnenstruktur gegeben werden.

$46 \mathrm{Vgl}$. Presseinformation "Recht $\%$ des Bundesministers der Justiz vom 14.4.1991.

47 So auch Frommel, M., ([.n. 37), S. 183 f. Caesar-Wolf, Beatrice/Eidmann, Dorothee: Gleichberechrigungsmodelle im neuen Scheidungsfolgenrecht und deren Umsetzung in die familiengerichtliche Praxis, in: Zeitschrift für Rechtssoziologie Heft 2/1985, S. 163-189; Berghahn-Wolffram, Sabine: Neue Chancen für geschicdene Frauen? Fine Untersuchung über die Rechtspraxis von Ehegateenunterhalt zur Qualifizierung. Franklurt a. M. u. a. 1992. 
3.1 Veränderungsvorschläge zum Verfassungsstatus von Ehe, Familie und anderen Lebensformen

In der öffentlich geführten Verfassungsdiskussion wurde eine Vielzahl von Formulierungsvorschlägen produziert, gerade von frauenpolitischer Seite. Nach den ersten Manifesten von "Frauen in bester Verfassung " und "Frauen für eine neue Verfassung (Irankfurter Frauenmanifest) in Jahre 1990 sind entsprechende Aktivitäten r99r in den kompromißhaften Entwurf des "Kuratoriums für einen demokratisch verfaßten Bund deutscher Länder « eingeflossen, der auch an ostdeutsche Vorschläge des Verfassungsentwurfs des Runden Tisches (1990) anknüpfte. In der Folgezeit wurden noch zahlreiche andere Vorschläge der Parteien in den Bundesländern, von Frauenorganisationen und anderen bürgerrechtlichen Gruppen erarbeitet und z. T. der Öffentlichkcit vorgestellt. ${ }^{+8} \mathrm{Im}$ Dezember 1992 fand dann einc öffentliche Anhörung vor der Gemeinsamen Verfassungskommission von Bundestag und Bundesrat statt, in der sechs ExpertInnen ihre Einschätzung zu bestimmten Fragen und z. T. eigene Formulierungsvorschläge vortrugen. Hier soll im folgenden schwerpunktmäBig auf diese Stellungnahmen eingegangen werden, soweit sie den Status von Ehe, Familie und anderen Lebensgemeinschaften oder -formen angehen.

Die beiden Gutachter Axel Freiherr von Campenhausen und Heinhard Steiger hatten es insofern leicht und konnten auf Formulierungsvorschläge verzichten, weil sie den bestehenden Verfassungsstatus von Ehe und Familie verteidigten. ${ }^{+9} \mathrm{Campen}-$ hausen tat dies mit einer bereits 1987 veröffentlichten Stellungnahme samt allen mit der Vorrangstellung der Ehe verbundenen Ausgrenzungen anderer »Gescllungsformen $4.5^{\circ}$ Er geißelte darin sogar die schleichende Aushöhlung des Eheschutzes unter dem geltenden Recht und bezeichnete diesen Zustand als "schreiende Mißachtung der Verfassung durch den Gesetzgeber ". "Stciger billigte seheähnlichen Lebensgemeinschaften«, wic sie das Bundesverfassungsgericht in seiner jüngsten Entscheidung vom 17.11. 1992 definiert hat, zu denen abcr z.B. glcichgeschlechtliche Zweierbeziehungen demnach nicht gehören (können), immerhin cine beschränkte Gleichstellbarkeit durch den Gesctzgeber zu, rechtfertigte ansonsten aber die institutionelle Garantie der Ehe mit der wbesonderen anthropologischen und gesell-

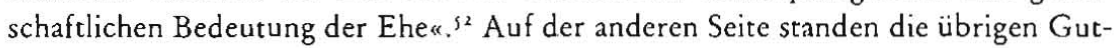
achterInnen, die mehr oder wcniger weitreichende Veränderungen an Art. 6 GG befürworteten, vor allem auch zum Rechtsverhältnis von Ehe, Familic und anderen Lebensgemeinschaften. Waren Schutz und Förderung der Ehe aus dem Entwurf des Kuratoriums und aus seinen feministischen Vorformen noch völlig verbannt worden,

${ }_{4} 8 \mathrm{Z}$. B. das Kuratorium: Vgl. Dokumentatıon der Anhorung des Verfassungskuratoriums $*$ Frauenrechte in der Verfassung* vom 15.5.1992 in Bonn.

49 Prof. Dr. Axel Fresherr von Campenhausen: Beibehaltung des geltenden Art. 6 Abs. 1 mit streng eheprivilegierender Auslegung, vgl. Campenhausen, Axel Freiherr von, (Fn. 9. 19)

Prof. Dr. Heinhard Steiger: keın Formulierungsvorschlag, inhaltlich Beibehaltung des geltenden Wortlauts mit folgender Auslegung: -1 . Die Ehe darf gegenuber der eheảhnlichen Gemeinschaft aufgrund des Art. 6 Abs. I GG nicht benachteiligt werden.

2. Die eheahnliche Gemeinschaft steht unter dem Schutz des Art. 2 Abs. I GG i. V.m. Art. 1 Abs. 1 GG (Personlichkeitsrecht); sie darf nıcht sim Úbermaß erschwert oder unmoglich gemacht werden.

3. Der Gesetzgeber kann die eheahnliche Gemeinschaft im sozialen Bereich mit der Ehe im Rahmen seines Gestaltungsspielraums wegen der Ahnlichkert mit der Ehe als sauf Dauer angelegte Verantwortungs- und Einstehungsgemeinschaft. gleichstellen. $\mathrm{V}_{\mathrm{gl}}$. schriftliche Stellungnahme, Ms.

so Campenhausen, A. Frciherr von, (Fn. 19), S. 17.

si Ebenda, S. 47

s2 Steiger, H., (Fn. 9), S. 3 . 
so wurden sie in fast alle neuere Formulierungsvorschläge zu Art.6 GG wieder aufgenommen, insbesondere auch in drei der vier innovationsorientierten Stellungnahmen vor der Gemeinsamen Verfassungskommission. Michael Coester behielt in seiner Stellungnahme zwar den Beziehungsschutz für dic Ehe wie gehabt bei, interpretierte aber schon den geltenden Art. 6 Abs. I GG weniger ehe-privilegierend, zudem propagierte er für »andere Lebensgemeinschaften « eine schwächerc verfassungsrechtlichc Garantie in Form der »Achtung«. Ute Gerhard und Eva Marie von Münch siedetten in ihren Formulierungen dagegen die Ehe auf einer Ebene mit anderen Lebensgemcinschaften und im Hinblick auf die Schutzintensität unterhalb der "Familie" an."3 Gebrauchte Formulierungen waren hier "Achtung u und "Schutz " für die Ehe und andere Lebensgemeinschaften, wobei letztere z. T. definitorisch eingeschränkt wurden auf "registrierte«, "auf Dauer angelegte (Gerhard) oder auf "verantwortet gclebte " (von Münch).

Fast alle Veränderungsvorschläge sind zum Begriff der "Iamilie zurückgekehrt. Dieser Rückgriff auf "Bewährtes" und lebenswcltlich positiv Besctztes hattc schon beim Kuratoriumsentwurf und beim Entwurf des Runden Tisches stattgefunden. In den beiden Frauenmanifesten hingegen wurde die institutionelle Auffassung kritisiert und in ein soziales Grundrecht umformuliert. Die förderungswürdigen familiären Konstellationen sind dort radikal konkret formuliert worden, z. B. als "Frauen und Männcr, die Kinder aufziehen, (Kranke oder alte Menschen versorgen) « ${ }^{54}$. Jutta Limbach machte zunächst den Vorschlag," zwar das Wort "Familie « beizubehalten, aber zusammen mit dem Zusatz »ungeachter des Familienstands ihrer Mitglieder « zu verwenden. Damit sollte ausgedrückt werden, daß nicht mehr allcin die eheliche Familie Definitionsgrundlage des neuen Grundrechts sein sollte. Auf diese Weise sollte das Familienverständnis vom institutionellen Ehebegriff gelöst und die ZweiFamilien-Akrobatik der herrschenden Meinung im Fall von nichtehelichen Paaren mit Kind (s. o.) abgeschaffe werden. ${ }^{36}$

Die Vorschläge der drei Gutachterinnen Ute Gerhard, Barbara Degen und Eva Marie von Münch zeugten von dem gemeinsamen feministischen Bemühen um eine Abschaffung oder Reduzierung der Privilegierung der Ehe und der institutionalisierenden Wirkung entsprechender Formulierungen. Das drückte sich vor allem darin aus, daß eine konkrete staatliche Schutz- und Förderungspflicht für Kindererziehung, Pflegetätigkeit und Erleichterungen zur Vercinbarung eben dieser Tätigkeiten mit Beruf und gesellschaftlicher Partizipation in einem weiteren Absatz ausdrücklich formuliert wurde. ${ }^{57}$ Durch dieses Zusammenspiel der Absätze wird die Rangfolge

53 Formulierungsvorschlage zu Art. 6 Abs. I, vgl. schriftliche Stellungnahmen:

Prof. Dr. Michael Coester: kein Formulierungsvorschlag, bekrátugt Bedeutung des Schutzes und der Forderung der Familie im weiteren Sinne, interpretiert Ehe schon jetzt als nicht mehr die Gleichstellung anderer Lebensgemeinschaften ausschließend, will in Zukunft zwar Eheschutz erhalten, aber s Achtung. wanderer Lebensgemeinschaften aufnehmen.

Barbara Degen: $\triangleright$ Der Staat achtet alle Lebensformen und schutzt sie vor Diskriminierung. ....

Prof. Dr. Ute Gerhard: -Der Staat achtet Ehe und Famılie und schutzt deren personlichen Lebensbereich. Das gleiche gilt fur andere registrierte (auf Dauer angelegte) Lebensgemeinschaften.*

Dr. Eva Marie von Munch: •Die Familie steht unter dem besonderen Schutz der staarlichen Ordnung. Die Ehe und andere verantwortet gelebte Lebensgemeinschaften genießen Achtung und Schutz. *

\$4 Frankfurter Frauenmanifest "Frauen fur eine neue Verfassung«, in: Femınistische Studien extra 1991, S. 111. Áhnlich: „Frauen in bester Verfassung $\alpha$, ebenda, S. 1 is

Ss Limbach, Jutta: Zur Rechtsstellung von Eltern und Kındern, in: Feministische Studien extra 1991, S. siff., si.

s6 Inzwischen hat sich auch Jutta Limbach - schon durch die Hartnackigkeit ihrer Parteifreunde - wieder zur Beibehaltung des Eheschutzes bekehren lassen, vgl. Dokumentation der Anhorung des Kuratoriums, (Fn. 48), S. 27 f.

57 Vgl. Kuratoriumsentwurf 2. Absatz, Verfassungsentwurf des Runden Tisches Art. 22 Abs. 2 Satz 2, Ute Gerhards Vorschlag als 2. Absatz, Eva Marie von Munchs Vorschlag für einen dritten Absatz, Barbara 
zwischen einer aktiven staatlichen Förderung der (funktionalen) Familie und dem lediglich auf Achtung und Schutz ausgerichteten Abwehrgrundrecht von Ehen und anderen Lebensgemeinschaften verdeutlicht.

Die einzige unter den GutachterInnen vor der Gemeinsamen Verfassungskommission, die nicht die Ehe und auch nicht die Familie in ihren Formulierungsvorschlag zu Art. 6 GG aufgenommen hat, war Barbara Degen, die in Abs. I dem Staat die Achtung aller Lebensformen, also auch der von Alleinstehenden und -lebenden, sowie deren Schutz vor Diskriminierung auferlegen wollte. Sie hat also den ursprünglichen feministischen Gedanken der radikalen Entprivilegierung der Ehe und der über diese definierten Familie beibehalten und sich sogar noch darum bemüht, die Möglichkeit der Institutionalisierung von Zweierbeziehungen überhaupt im Ansatz zu unterbinden. Im zweiten Absatz werden - ähnlich wie bei Ute Gerhard konkret Menschen, die Kinder erziehen odcr Hilfsbedürftige betreuen, mit einem Schutz- und Förderungsanspruch versehen. Auf diese Weise ist der Begriff "Familie" als möglicher Ansatzpunkt für die Reinstitutionalisierung der Ehe "durch die Hintertür« ausgeschaltet. Degen griff damit sowie mit der Berücksichtigung »aller Lebensformen « eine sehr grundsätzliche Kritik auf, die auch den meisten Neuformulierungsvorschlägen entgegengehalten wird, nämlich das Prinzip der Institutionalisierung und Privilegierung bestimmter Formen des Zusammenlebens beizubehalten und dadurch wiederum herausfallende Lebensformen, wie z.B. die von Alleinlebenden, zu diskriminieren. ${ }^{8}$ Auch Ute Gerhard plädicrte für eine Loslösung des Familienbegriffs von der Vorstellung des gemeinsamen Wohnens bzw. des gemeinsamen Haushalts, denn diese entspreche soziologisch längst nicht mehr der Realität. Es gebe »haushaltsüberschreitende Familiennetze und Solidarbeziehungen, die psychische und materielle Unterstützung und Betreuung bieten. In diesem Sinne könnte, Gerhard zufolge, der erweiterte Iamilienbegriff "sich bewußt von der Vorstellung gemeinsamen Wohnens und Wirtschaftens (nur mit ledigen Kindern) lösen und die sozialen Beziehungen umfassen, insbesondere die gegenseitige Hilfe und Anteilnahme nicht nur zwischen den Generationen sondern auch zwischen Personen, die sich als Lebensgemeinschaft definieren. "s9

\subsection{Was ist verfassungsrechtlich beute noch schutz- und förderungswürdig?}

\subsection{Sicherlich nicht in erster Linie die Institution Ebe!}

Es versteht sich nach alledem fast von selbst, daß der institutionelle und privilegierende Eheschutz und die entsprechende Förderung verfassungsrechtlich mit einer modernen pluralistischen Gesellschafts- und Rechtsauffassung nicht vereinbar sind. Die Versuche, den privilegierten verfassungsrechtlichen Ehestatus aus einem anthropologischen Bedürfnis der Menschheit zu konstruieren (s.o.), beruhen auf einer kaum verhüllten Unlogik. Denn es mag sicherlich ein in der Menschheitsentwicklung bisher konstant vorhandenes Bedürfnis nach Zweierbeziehungen geben, der

Degens Vorschlag für einen 2. Absatz. Beispielhaft hier Ute Gerhards Vorschlag: „Wer in familiarer Gemeinschaft Kinder erzieht oder für andere sorgt, iss durch die staatliche Ordnung zu schurzen und zu fördern. Der Staa fördert die Moglichkeiten von Eltern und Alleinerziehenden, ihre Frziehungsaufgaben mit der Erwerbstatigkeit und der Teilnahme am offentlichen Leben zu vereinbaren. $\propto$

s8 Degen, (Fn. 9), S. 4 f.; ahnlich Frommel, (Fn. 37), vgl. Anm. 42, S. 187.

s9 Gerhard, U., (Fn.9), S.6, in $6 \mathrm{f}$ 
spezifischen Rechtsform wohnt aber - selbst von den meisten Konservativen zugegeben - keine anthropologische Gesetzlichkeit, nicht einmal überhistorische Kontinuität inne. ${ }^{60} \mathrm{Um}$ die Privilegierung der Ehe zu rechtfertigen, muß also von Konservativen auf vorrangige ordnungspolitische Staatsinteressen oder doch zumindest auf eine angeblich überlegene Zweckmäßigkeit der Ehe zur Regelung des Zusammenlebens und der Familienbildung zurückgegriffen werden. So schreibt etwa von Campenhausen:

"Der Grund (für den verfassungsrechtlichen Schutz der Ehe/Ergänz. d. Verf. S. B.) ist vielmehr dic einzigartige Leistung der Ehe als sittlich-personaler Lebensbeziehung und ihre daraus folgende öffentliche Funktion. Die vorbehaltlose Zusage der Ehegatten bedingt die monogame Struktur der Ehe, welche als Subjekt-Subjekt-Beziehung Ausdruck eines Ethos der Menschenwürde ist, die nicht nur Teilaspekte des anderen will, sondern den ganzen Menschen als Menschen annimmt. Dies ist auch die Grundlage für eine Gleichbcrechtigung der Geschlechter. Nur die Ehe besitzt die Anlage auf Lebensdauer und die Offenheir für das Kind. " ${ }^{61}$

Hier zeigen sich aber deutlich die Schwachstellen der Argumentation: Kann eine christlich-religiöse Annahme über Sittlichkeit und Ethos in einem säkularisierten Gemeinwesen ohne weiteres verallgemeinert werden? Woher nimmt Campenhausen die empirische Gewißheit, daß in Ehen eher eine vorbehalt tose Annahme des anderen stattfindet, eher Gleichberechtigung, Dauerhaftigkeit und Offenheit für das Kind existieren, wobei mit letzterem vermutlich doch nur die Bereitschaft zum Kinderbekommen gemeint ist, die persönliche Haltung gegenüber dem realen Kind nicht einmal tangiert wird? Die ordnungspolitische Variante läßt sich also in dieser Pauschalität und mit den gewünschten Ausgrenzungen nur in einer autoritär strukturiercen "formierten" Gesellschaft begründen, in der es dann konsequenterweise aber auch weiterhin möglich sein müßte, nichteheliches Zusammenleben und Kindererziehen zu unterbinden. Wo Demokratie und Privatautonomie ernst genommen

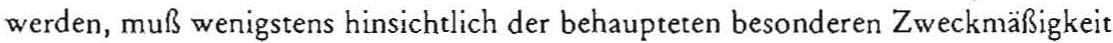
der Ehe der Nachweis einer so optimalen Funktionserfüllung erbracht werden, daß die Einschränkung der Selbstbestimmung der Gesellschaftsmitglieder legitim erscheint. Denn nur weil die Institution Ehe rechtspolitisch bequem für den Staat ist und dieser für die Individuen "nur das Beste will", braucht eine so eingreifende Konstruktion noch lange nicht gerechtfertigt zu sein. Die Ungleichbehandlung von Individuen und Lebensgemeinschaften, insbesondere aber faktisch der Geschlechter spricht eher dagegen. Konservative wie Steiger und von Campenhausen gehen jedoch über alle sattsam bekannten empirischen Funktionsdefizite der Ehe und normativen Abwägungserfordernisse hinweg. Von Campenhausen behauptet sogar, daß die Ehe ihre Funktion deshalb nicht erfüllen könne, weil sie vom Gesetzgeber und der Rechtsprechung nicht streng genug ausgrenzend exekutiert werde. ${ }^{62}$ Bei alledem verharren die Verteidiger der privilegierten Ehe bei einer pauschalen und weiterhin vorrangig institutionellen Betrachtung und setzen Familie mit Ehe im Ansatz gleich, als ob beide nicht längst in unterschiedliche Funktionszusammenhänge zerfallen wären. Es ist also ein Zirkelschluß, die besondere Angemessenheit der Ehe mit ihren erwünschten, aber nicht tatsächlichen Eigenschaften für die Gesellschaft und die Individuen begründen zu wollen. Es ist zudem eine autoritäre Bevormundung einer

60 Zum Bedeutungs- und Gestaltungswandel der Ehe vgl. Sticgler, B. , (Fn. 9), S. $132 \mathrm{ff}$.

61 Campenhausen, A. Freiherr von, (Fn. 19), S. 18; ahnlich auch Steiger, H.: $\bowtie$ Maßgebend ... ist die besondere anthropologische und gescllschaftliche Bedeutung der Ehe. Sie stellt eine allgemeine Grundstruktur der personalen Lebensgestaltung und -ordnung von Mann und Frau aber auch fur die Gesellschafi dar. Sie hat sich zwar im Laufe der Geschichte inmer wieder grundlegend gewandelt, auch in den letzten 30 Jahren wieder. Aber als besondere, rechelich geformte, institutionalisierte Gestaltungsform hat sie sich gerade auch durch ihren Wandel erhalten." Stciger, (Fn. 9), S. 129.

62 Campenhausen, A. Freiherr von, (Fn. 19), S. 29 ff. 
wachsenden Zahl von Personen, die die Ehe für sich als keineswegs angemessen ansieht, aber dennoch bedeutende soziale und gesellschaftliche Leistungen erbringt.

\subsubsection{Grundrechtsschutz vor allem für die soziale Dimension der Familie!}

War eine Streichung der Ele aus Art. 6 Abs. r von vornherein ohnehin nicht realistisch, so lag es für die ReformerInnen nahe, sich auch auf der Forderungsebene erst einmal darauf zu besclaränken, zwischen dem Grundrechtsgegenstand der Familic und dem der Ehe bzw. Zweierbeziehung zu unterscheiden. Dabei dürfte es einleuchten, daß nur die "Familie «, d. h. das Kindererziehen und die Pflege hilfsbedürftiger Menschen, den vollen Grundrechtsschutz in abwehr- und teilhaberechtlicher, d.h. sozialer Hinsicht verdient. Die Rechts- und Sozialordnung müßte daraufhin durchgeforstet werden, wie diese soziale Fördcrung besser und ohne Privilegierung der Ehe gewährleistet werden kann. Für die in Ehen und nichtehelichon Gemeinschaften realisierte Beziehung bliebe dann allenfalls noch ein abwehrrechtlicher Schutzanspruch, der sich auf erheblich bestandsgefährdende staatliche Eingriffe beziehen könnte. Ehe und nichtcheliche Beziehung wären konsequenterwcise dann auf die gleiche Stufe zu stellen. Die Institution Ehe verlörc so beträchtlich an Verfassungsrang. Schon deshalb waren Formulierungsvorschläge diesen Inhalts in der Verfassungskommission nicht mehrheitsfähig. Als Kompromiß wurde daher in einigen Vorschlägen zwischen dem vollen "Schutz " der Ehe und einer "Achtung " nichtehclicher Gemeinschaften unterschieden. ${ }^{63}$ Dies würde jedoch eine Reihe ncuer und alter Differenzierungsprobleme mit sich bringen, ohne sic zu lösen, entsprach insofern aber ciner realistischen Einschätzung der Widerstände gegen die konsequente Entprivilegierung der Ehe.

Nüchtern und funktional betrachter liegt es jcdenfalls auf der Hand, daß in erster Linie die Familienfunktion in bezug auf Kinder und Pflegebedürftige schützens- und förderungswürdig ist. Allerdings ist bei der Verwendung des Wortes »Familie Vorsicht gebotcn, weil dabei allzu leicht die Kontinuität der bisher herrschenden Interpretation als »ehelicher * Familic crhalten bleibt (s.o.). Im Hinblick auf dic ersten radikaleren feministischen Formulierungen, in denen eine verfassungsrechtliche Thematisierung der reinen Beziehungsebenc (ohne Kinder oder andere Sozialfunktionen) ganz unterblicben war, stellt sich die Frage, ob nicht doch das bisherigc Abwehrrecht der Ehe - nunmehr aber für eheliche und nichtehcliche Zweierbeziehungen - wciterhin vonnötcn sein könnte, um gegen erbeblich bestandsgefährdendc staatliche Eingriffe zu schützen. Beispiele für derartige Gefahren finden sich im Ausländer-, Arbeits-, Sozialrecht oder Strafrecht.

\subsubsection{Daneben Grundrechtsschutz auch für die (kinderlose) Zweierbeziehung?}

Sollte sich nämlich die Individualisierung auch in der Rechtssphäre soweit durchsetzen, daß der Rekurs auf die Gemeinschaft der Ehegatten, Lebenspartner oder der näheren Verwandten unterbunden oder zurückgeschraubt wird, so bedeutet dies, daß die individucllen Erwerbs-, Mobilitäts- und Selbstbehauptungsanfordcrungen erhöht werden; dieser Trend ist bereits zu beobachten. Dic Aufrechterhaltung und -haltbarkeit von engen Bezichungen ist angesichts enorm gesticgener Mobilitätsanforderungen in manchen Berufsgruppen oder Regionen schon jetzt zum persönli- 
chen Risiko geworden, ${ }^{64}$ was sich bislang - jedenfalls für Frauen - eher für die Aufgabe von Bindungen behaupten ließ. Die Aufrechterhaltung von Beziehungen wird auf diese Weise zunehmend dilemmatisch für die eigene Selbstbestimmung und eine eigenständige ökonomische Existenz. Sollte sich neben diesen sozialen Veränderungen auch die Verfassungslage in Richtung auf eine Ausblendung der Zweierbeziehung aus Art. 6 ändern, was nun allerdings erst einmal ausgeschlossen ist, wäre es für die staatliche Gesetzgebung und Rechtsprechung längerfristig kaum mehr plausibel, allzu viel Rücksicht auf das Bindungsinteresse von Erwachsenen ohne Kinder (oder Pflegebedürftige) zu nehmen, wo der Staat doch aus unterschiedlichen Gründen, insbesondere aber bei knappen finanz- und sozialpolitischen Ressourcen, unter enormen Handlungs- und Differenzierungsdruck steht. So hat z. B. im Ausländerrecht selbst die geltende "Heiligkeit" der Ehe nicht verhindert, daß das Recht auf Familiennachzug und der Schutz vor Ausweisung inzwischen stark relativiert sind. ${ }^{6}$ s

Um sozusagen das "Äußerste an Individualisierung im Recht zu verhindern, würde - unter der Bedingung der Umwandlung von Art. 6 Abs. I GG in ein soziales Familiengrundrecht - ein daneben weiterexistierendes Abwehrrecht der Zweierbeziehung gegen deutlich bestandsgefährdende staatliche Eingriffe denjenigen entgegenkommen, dic einen Kernbereich privatautonomer Interpersonalität schützen wollen. Konservative würden sicherlich versuchen, dieses Recht weiterhin eheprivilegierend zu interpretieren und dabei vor allem auf den Effekt der Geburtenförderung schielen. Diese Gefahr kann aber kein ernsthaftes Gegenargument sein, denn der ausgrenzende Anschluß an das Rechtskonstrukt Ehe wäre ihnen genommen, und die Reduzierung auf ein Abwehrrecht ließe sich nicht so leicht ignorieren. Linksliberalen Kritikern der Verrechtlichung und "Kolonialisierung " von verständigungsorientierter Lebenswelt ${ }^{66}$ müßte ein solches reduziertes "Kerngrundrecht " ebenfalls am Herzen liegen. Letzterc diagnostizieren schon jetzt verstärkte Abhängigkeit, hervorgerufen durch ein Netziverk von staatlichen Interventionen im Familienrecht. Auch eine Überfrachtung des Einzelnen mit Individualisierungsrisiken und der völlige Verzicht auf einen Schutz der Möglichkeiten zur Beziehungsaufrechterhaltung könnten so das "Projekt Selbstbestimmung ${ }^{{ }^{67}}$ gefährden.

\subsubsection{Individualgrundrechte versus Beziehungsgrundrecht}

Im Gegensatz zu dieser Argumentation für ein rudimentäres, nämlich nur noch auf Abwehr angelegtes Beziehungsgrundrecht vertritt Monika Frommel die Auffassung, daß der Schutz der Zweierbeziehung statt über Art. 6 GG über den Würdebegriff (Art. r Abs. I GG) oder die allgemeine Handlungsfreiheit (Art. 2 Abs. I GG) oder über eine Verbindung beider besser zu gewährleisten sei. ${ }^{68}$ Frommel möchte die

64 Beck, Ulrich/Beck-Gernsheim, Elisabeth: Das ganz normale Chaos der Liebe. Frankfurt a. M. 1990, S. 190 .

6s Geht es um eine Aufenthaltsgenehmigung fur cinen auslandischen T.hegatten einer inlandischen Person, so muß zwischen dem Schutzgebot von Art. 6 Abs. I GG cincrscits und einer Beeintrachtigung der Belange der Bundesrepublık Deutschland abgewogen werden, das Schutzgebor begrenzt dabei das eingersumte Ermessen bzw. muß schon bei der Auslegung der Rechtsbegriffe berücksichtigt werden, vgl. Maunz/Durı/Herzog, (Fn. 7), Anm. Il 4 c) zu Art. 6 GG. Fur den Familiennachzug von Ausländern gilt ein nochmals reduzierter Familienschutz, obwohl Art. 6 Abs. I GG ein Menschenreche ist; ublicherweise gibt es mehrjahrige Wartefristen fur Ehegatten bzw. Alters:renzen fur Kinder.

66 Habermas, Jürgen: Theorie des kommunikativen Handelns, Frankfurt a. M. 1981, Bd. 2, S. $522 / 523$

67 Simitıs, Spiros: Selbstbestimmung: Illusorisches Projekt oder reale Chance?, in: Kritısche Justiz, Heft $1 / 1988, S_{3} 32-50$

68 Auch der Gutachter Steiger, (Fn. 9), S. 131 f., und das Bundesverfassungsgericht in seiner Entscheidung vom 17. 11. 1992, Az. 1 BvL 8/87, S. sof., subsumieren den Anspruch der nichtehelichen Gemeinschaft, 
individuellen Grundrechte auch relationistisch verstanden wissen, in dem Sinne, daß jeder Mensch in Relation zu anderen Menschen steht und Grundrechte daher auch diese Bindungsdimension einbeziehen. Dennoch erscheint es fraglich, zum einen, ob sich eine solche Auffassung in der Verfassungsinterpretation durchsetzen würde bisher wurde sie entgegen der Auffassung von Steiger ${ }^{69}$ jedenfalls nicht praktiziert -, und zum anderen, ob sie zu sinnvollen und sozialadäquaten Ergebnissen führen würde. Ein Beziehungsschutz gegründet auf Art. I und 2 GG könnte sicherlich eklatant inhumane staatliche Kontakt- oder Beziehungsverbote wie etwa im Nationalsozialismus abwehren, ansonsten fehlt es aber am spezifischen Regelungszusammenhang, aus dem sich Kriterien ableiten ließen, die beschreiben können, welche Beziehungen vor welchen staatlichen Eingriffen geschützt werden sollen. Die herkömmliche Zweierbeziehung, sei sie ehelich oder nicht, ist jedoch eine sehr spezifische zwischenmenschliche Verbindung, deren Abgrenzung von andcren Sachverhalten insofern durchaus cin Minimum an Institutionalisierung, d.h. Auf-DaucrStellung und normative Heraushebung erfordert. Im Zusammenhang von Menschenwürde und Handlungsfreiheit wäre dagegen entweder jede relationale Identität stiftende Beziehung beispielsweise gegen staatlich verordnete Mobilitätserfordernisse im Arbeitslosenrecht zu schützen, z. B. die einer Person zu drei Geliebten gleichzeitig, zu den liebsten Arbeitskolleginnen oder zu den drei Katzen der Nachbarschaft; oder aber keine dieser sozialen "Normalbezichungen «, zu denen - wenn auch an prominenter Stelle - die ehcliche oder nichteheliche Zweierbeziehung gehört, fiele unter den Schutz, weil es weder inhuman ist, noch den Kernbereich der ohnehin unter Gesetzesvorbehalt stehenden allgemeinen Handlungsfreiheit tangiert, wenn man/frau eine "Spagatbeziehung "führen müßte, um nicht arbeitslos zu werden oder zu bleiben. Wer die beschriebenen Auslegungs- und Auslegungsdurchsetzungsprobleme vermeiden will, ein Abwehrrecht der Zweierbeziehung aber befürwortct, sollte daher versuchen, eine eindeutige Formulierung im mindestens historisch dafür einschlägigen Regelungszusammenhang des Art. 6 GG zu treffen.

\subsubsection{Feministische Einwände gegen überböhte Autonomievorstellungen}

Bci Monika Frommels Einwänden gegen ein rudimentäres Beziehungsgrundrecht gcht es um das Spannungsverhältnis von wünschenswerter individueller Autonomie und den Gefahren von institutionalisicrten und letztlich privilcgierten Beziehungsformen. Frommel vertritt dabei den radikal-liberalen Standpunkt weitestgehender individueller Autonomie. Andere FeministInnen zielen dagegen im Theoriediskurs anders als in der hiesigen Verfassungsdiskussion - oftmals in die entgegengesetzte Richtung, nämlich gegen die prioritärc Vorstellung von Autonomie und Unabhängigkeit, die als "männlich « idealisiert kritisiert werden..$^{70}$ Autonomie und Unabhängigkeit setzen demnach stillschweigend voraus, daß cine andere (abhängige) Pcrsonengruppe den scheinbar Unabhängigen (und somit für drittc Instanzen wic z. B. den Arbeitsmarkt frei Verfügbaren) den Rücken freihält. Auf diese Weise sind die Kontextgebundenheiten an Beziehungen, Kindererziehung, Kranken- oder A]-

\footnotetext{
als Lebensform geduldet zu werden, also nicht erwa wim Übermaßa erschwert oder unmoglich gemache zu wcrden, unter Ar. 2 Abs. i (i. V.m. Arr. 1 Abs. 1) GG. Dic Schutzintensitat ist jedoch deutlich geringer angelegt als bei der Ehe. Die gesetzliche Behandlung nichtehelicher Gefahren im Ausländerrecht oder im Hinblick auf das Zeugnisverweigerungsrecht im Strafprozeß sprechen gegen die Effektivitat einer solchen Auffanginterpretation von Art. 2 Abs. I GG.

$69 \mathrm{Vgl}$. vorherige Anm.

70 Als Übcrblick vgl. Baer, Susanne/Berghahn, Sabine: A uf dem Weg zu einer feministischen Rechtskultur? Deutsche und US-amerikanische Ansarze, in: Berliner Policologinnen (Hg.): Femtnistısche Standpunkte in der Politikwissenschaft. Darmstadt, erscheint voraussichtlich 1994.
} 
tenpflege und Alltagsverrichtungen in dieser Gesellschaft durchaus geschlechtsspezifisch unterschiedlich intensiv, wenn auch oft nur im Bewußtsein. Bis in die Theorien von Ethik und Gerechtigkeit zieht sich die bekannte Kontroverse um eine geschlechtsspezifische Moral (Gerechtigkeitsprinzipien versus Fürsorge?) bzw. um die Orientierung am abstrakten oder am konkreten Anderen." Für das Recht ist daher wiederum von feministischer Seite auch die Anforderung gestellt, daß es auf die sozialtypischen Kontextgebundenheiten von Menschen so Rücksicht nimmt, daß Rollenstereotypen weder verfestigt werden, noch fürsorgliche Verhaltensweisen als autonomieabträglich unterbunden werden. Dazu müßten eben wwesentliche" Kontextbindungen an Kinder, Pflegebedürftige und Beziehungspartner im Grundsatz auch vom Verfassungsrecht für Männer und Frauen universalisiert werden. Die alternativen Vorschläge sind dem bezüglich der Sorge für Kinder und Pflegebedürftige durch die Etablierung eines prioritären und konkret formulierten sozialen Schutzund Teilhaberechts nachgekommen. Es bleibt die Frage, ob und in welcher Form auch enge Beziehungen unter Erwachsenen wenigstens eine abwehrrechtliche Bestandsgarantie genießen sollten.

Die Forderung danach könnte aber erneut feministische Bedenken auf den Plan rufen, weil schon der geltende abwehrrechtliche Schutz der Ehe einen eher doppelbödigen Umgang mit der Selbstbestimmung der »Partner «bzw. Familienmitglieder aufweist. In der Tat ist zu kritisieren, daß der Staat sich oft opportunistisch gerade dann mit Interventionen zurückhält, wenn ein Eingreifen zum Schutz der Schwächeren, meist Frauen und Kinder, notwendig ist (Gewalt in Ehen und Beziehungen, Vergewaltigung, Kindesmißhandlung, sexueller Mißbrauch), und wenn die Selbstbestimmung von einzelnen gerade nicht gegeben ist (z.B. Zwang der Banken zur Mithaftung der erwerbslosen Frau für Kredite an den Ehemann). Die Respektierung der abgespaltenen "Intimsphäre « ${ }^{72}$ des Paares bzw. der Familie durch den Staat droht so zum Mythos zu werden, der vor allem die Erhaltung patriarchaler Gewalt- und Ausbeutungsverhältnisse verschlejert. ${ }^{73}$ Aus dieser feministischen Kritik an der bürgerlich-liberalen Familienauffassung ergibt sich in dem hier diskutierten Zusammenhang aber kein grundsätzliches Gegenargument. Denn die z.T. augenfällige Diskrepanz zwischen Anspruch und Wirklichkeit kann nicht dazu führen, dem Staat den totalen Zugriff zu ermöglichen. Schon aus logischen Gründen setzt das Abwehrrecht voraus, daß die Beteiligten ihre Beziehung einvernehmlich geschützt und aufrechterhalten sehen wollen.

\subsubsection{Diskriminiert ein rudimentärer Beziehungsschutz Alleinlebende?}

Barbara Degens Formulierungssorschlag und Monika Frommels Bedenken richten sich gegen den Versuch, weiterhin bestimmte Zweierbeziehungen, nämlich Ehen und "auf Dauer angelegte " nichteheliche Gemeinschaften, ja überhaupt Zweierbeziehungen von Zusammenlebenden mit Verfassungsrang auszustatten, wcil dics zur Diskriminierung anderer Lebensformen, speziell der Alleinlebenden führen könnte. Barbara Degen möchte deshalb einen Schutzanspruch waller Lebensformen a verankert sehen. Dem ist jedoch entgegenzuhalten, daß eine solche schutzrechtliche Gleichbehandlung von Beziehungen und Individuen schlecht möglich ist, weil sonst

7r Vgl. Benhabib, Seyla: Der verallgemennerte und der konkrete Andere. Ansaitze zu viner keministischen Moraltheorie, in: List, Elisabeth/Sruder, Herlinde (Hg.): Denkverhältnisse - Feminismus und Kritik. Frankfurt a. M. 1989, S. 454 ff.

72 Simitis, Spiros: Familienstruktur und Familienverfassung, in: Derselbe/Zenz, Gisela (Hg.): Seminar: Familie und Familienrecht. Bd. r, Frankfurt a. M. 1975, S. 121.

73 Olsen, F. E., (Fn. 36) 
die Konturen des Anspruchs verwischt werden. Wohlgemerkt soll es bei dem rudimentären Schutzanspruch der Zweierbeziehung - anders als beim sozialen Grundrecht der Familie - nicht mehr um die privilegierte Förderung wie heute noch bei der Ehe gehen, sondern lediglich um die Abwchr wesentlich bestandsgefährdender staatlicher Eingriffe. Wo keine feste Beziehung besteht, wäre dieser Anspruch sinnlos bzw. bei allzu lockeren Beziehungen sozial nicht gerechtfertigt, weil im Fall der staatlich veranlaßten Aufgabe der Beziehung wohl i. a. keine wesentlichen Nachteile auftreten. Wenn aber alle Lebensformen - und seien sie noch so verschieden gleich bchandelt werden sollen, tritt wieder ein ähnliches Problem auf wie mit der Ableitung eines Abwehrrechts der Beziehung aus Art. I und 2 GG, denn wenn der Gleichbehandlungsanspruch zu viele verschiedene Lebenssachverhalte betrifft, sinkt die Schutzintensität eines Abwehrrechtes. Es läßt sich nicht alles gleich behandeln, denn was gleich oder gleichwertig ist, läßt sich wohl nur durch die "Hinsichten« definieren, nach denen es gleich oder gleichwertig sein soll.

\subsection{7 list jegliche Institutionalisierung überhaupt vermeidbar?}

Bei der Zweierbczichung geht cs hier sicherlich um den Grad der emotionalen und praktischen Gebundenheit. Diese mit Kriterien zu messen und den Schutz in diesem Sinne zu operationalisieren, ist allerdings eine kaum zu bewältigende Aufgabe. Nicht umsonst verlangt das geltende Recht daher den formalen Akt der Eheschließung. Bei nichtehelichen Sachverhalten müßte er zum Zwecke der Abgrenzung erfaßter von nicht-erfaßten Sachverhalten ersetzt werden. Diese Abgrenzung aber bedeutet auch Ausgrenzung, sie stellt bestimmte Beziehungs- und Lebensformen auf Dauer, indem sie diese durch den Schutz - und sei er auch noch so rudimentär - attraktiver macht, als ancrkannt und empfehlenswert hinstellt und damit normativ heraushebt. All dies bedeutet Institutionalisierung, wenn auch in deutlich geringerem Umfang als bei der Ehe. Vermeidbar wäre sie nur mit einem konsequent individualistischen Grundrechtsverständnis, das unter (nicht-pflegebedürftigen) Erwachsenen überhaupt keinen Schutzanspruch gegen den Staat zur Aufrechterhaltung der Beziehung vorsieht.

Läßt man/frau sich aber auf derartige Institutionalisierungen ein, so wären allerlei Differenzierungen zu treffen. Ute Gerhard möchte nicht an das Zusammen-Wohnen anknüpfen, sondern an die einverständlich definierte Solidarbeziehung, die aber zum Beweis den Wcg der Registrierung gegangen ist, möglicherweise auch die Anforderung erfüllen sollte, wauf Dauer angclegt « zu sein (s, o.). Diese Anforderung würde dann rechtlicherseits vermutlich als Registrierungsvoraussetzung festgelegt werden und als eine Art Versprechen gedcutet werden, zusammen zu blciben. Würde die Registrierung als Fcststellungskriterium gewählt, so wäre ein notwendiges Zusammenleben entbehrlich. Das wärc zumindest praktikabel und ließe denen die Freiheit, die sich nicht derart binden wollen. Als Vorgabe für die Art und Weise der Institutionalisierung weniger sinvoll erscheint die Formulierung von Eva Marie von Münch zur "verantwortet gelebten Lebensgemeinschaft", denn wer sollte dies von welchem Zeitpunkt aus mit welchen Kriterien beurteilen können? Zudem gilt diese Anforderung nicht einmal für die Ehe. Des weiteren stellt sich die Frage, wieviele Beziehungen einer Person mit anderen, nicht verwandten Personen auf diesc Weise geschützt werden sollen. Trotz aller erfolgten Säkularisierungs- und Pluralisierungstendenzen bei den Lebensformen ${ }^{74}$ läßt sich wohl durchaus gesellschaft]ich konsensuell eine

74 Vgl. Nave-Hcrz, Rosemarie: Konunuitat und Wandel von Ehe und Familie, in: Dieselbe (Hg.): Wandel und Kontinuitat der Familie in der Bundesrepublik Deutschland, Stuttgart 1988, S.61-94. Als Überblick 
Eingrenzung auf jeweils eine geschützte Zweierbeziehung der daran Beteiligten vornchmen, aber keine Eingrenzung auf verschiedengeschlechrliche Personen rechtfertigen. Auch schwule und lesbische Paare sollten also an diesem Bestandsschutz für Beziehungen teilhabcn können. Die weitergehende Frage, ob ihnen die förmliche Eheschließung75 ermöglicht wcrden soll, ist, da sich eine Verknüpfung der Ehe mir Fortpflanzungserwartungen aus einer liberalen Grundrechrsperspektive von selbst verbieter, positiv zu bcantworten.

Realpolitisch betrachtet werden Lesben und Homosexuelle in Deutschland ${ }^{76}$ nicht die Möglichkeit zur förmlichen Heirat haben. Der jüngste Abweisungsbeschluß des Bundesverfassungsgerichts, mit dem die Drcier-Kammer des ז. Senats die Verfassungsbeschwerdc eines heiratswilligen homosexuellen Paares abwies, ${ }^{77}$ bestätige diese Annahme. Die Hauptbegründung des Gerichts, daß nach ständiger Rechtsprechung des BVerfG zu Art. 6 Abs. I GG nur die Gemeinschaft von Mann und Frau geschützt werde, weil die Vcrschiedengeschlechtlichkcit das Prägende an eincr Ehe sei, ist ersichtlich ein Zirkelschluß, zumal im weiteren gerade abgelehnt wird, die Fortpflanzungs(un)fähigkeit der Partner zum entscheidenden Kriterium zu machen. Als Funktion der Ehe wird in dem Beschluß lediglich die Absicherung der Partner im Falle einer Familiengründung ausgewiesen. Letztlich fehlt es so aber an einer Geltungsbegründung für die rcchtlichen Schutz- und Förderungswirkungen der Ehe für bewußt kinderlose Paare sowie an einer Rechtfertigung für die generelle Diskriminierung nicht-verheirateter Paare. Da dic Kammer letztcres offenbar selbst sieht, regt sie den Gesctzgeber zu einer Überprüfung einzelner Rechrsbereiche an. Somit spricht einiges dafür, daß sowohl nichtehcliche hetero- als auch homosexuelle Paare zukünftig durch eine leichte Nivellierung der Unterschiede der Nicht-Ehe zur Ehc einen kleinen Rechtszuwachs erhalten, möglicherweise aber nur nach Registrierung. Letztere mag den einen als der Ehc angenäherte Rechrsposition und damit positiv erscheinen, andere schen sie dagegen schon wegen der Vorenthaltung von Adoptionsmöglichkeiten in bezug auf Kindcr und wegen der im Registrierungsakt liegenden Stigmatisierungsgefahren als neue Diskriminierungsvariante..$^{8}$

Im Anschluß an dic Frage nach den durch ein rudimentäres Beziehungsgrundrecht geschützten Konstellationen wäre noch zu klären, wic intensiv eine von staatlichen Stcllen ausgehendc Bestandsgefährdung scin muß, um mit Hilfe des Schutzanspruchs abgewehrt zu werden. Diese Konkretisierung bliebe weitgehend der Gesetzgebung und vor allem Rechtsprechung übcrlassen.

Spätestens jetzt fällt auf, daß cin solch rudimentäres Abwchrgrundrccht für die Zweierbeziehung nicht gerade eine Neuheit wäre, sondern sich am Modell des bisherigen Eheschutzes, wenn auch nun in ausgedehnter Form, orientiert. Das ist jedoch kein Wunder, denn diese Veränderung wäre denn auch der größte und zugleich kleinste gemeinsame Nenner von Tradition und Modernität. Für die Gleichstellung von Frauen wäre schon mit der Entprivilegierung der Ehe viel gewonnen,

uber Diagnosen der post-modernen Familie vgl. Honneth, Axel: Zum Wandel familialer Lebensformen. Soziologie. Eine Kolumne, in: Merkur, Heft 1/1993, S. 59-64.

$75 \mathrm{Vgl}$ aber die spektakuliren Aufgebotsbestellungen von Hella von Sinnen/C.ornelia Scheel und vielen anderen im Jahre 1992, entsprechende Verfassungsbeschwerden und Argumente fur die schwul-lesbische Ehe, z. B. Bruns, Manfred/Beck, Volker: Die Ehe fur Lesben und Schwule aus rechtspolitischer Sicht, in: Zeitschrift für Sexualforschung, Heft $3 / 1991$, S. 192-204.

$76 \mathrm{Zu}$ Danemark vgl. Bech, Henning: Recht fertigen. Über die Einfuhrung shomosexueller Ehen * in Danemark, in: Zeitschrift fur Sexualforschung, Heft 3/1991, S. 213-224.

77 Beschluß des BVerfG vom 13. 10. 1993 (1BvR 640/93). Frankfurter Rundschau vom 14. 10.93.

$78 \mathrm{Vgt}$. Oesterle-Schwerin, Jutta: Assimilation oder Emanzipation? in: Zeitschrift fur Sexualforschung, Heft $3 / 1991$, S. $205-212$. 
weil die Lebenslüge der für Frauen angeblich paßgerechten Ehe allmällich ihre Täuschungswirkung ganz verlöre; zudem gäbc sic Anstöße zur Veränderung rechtlicher Statuskonstruktionen, z.B. in der Renten- oder Arbeitslosenversicherung, die Frauen heute noch benachteiligen und für die die (privilegierte) Ehe als Rechtfertigung herangezogen wird.

4. Ausblick

Die offizielle Verfassungsdebatte in der Gemeinsamen Kommission von Bundesrat und Bundestag ist ohne Änderungsvorschlag für Art. 6 GG zu Ende gegangen. Die Ehe wird wohl weiter privilegiert bleiben, das soziale Grundrecht der Familie kann schon aus diesem Grund vermutlich auch in Zukunft kaum Wirkung entfalten. Andere Lebensgemeinschaften müssen bis auf weiteres draußen bleiben. Der Wortlaut von Art. 6 Abs. I GG wird auf diese Weise zwar auch in Zukunlt eine relativ starre Hürde für einfachgesetzliche Alternativkonzeptionen und/oder gesellschaftsautonomen sozialen Wandel darstellen, es besteht jedoch die Hoffnung, daß im Zusammenwirken verschiedencr Tendenzen eine Entprivilegierung der Ehe im Verhältnis zur Familie und damit eine versachlichte und sozialere Interpretation des Grundrechts auch ohne Verfassungsänderung langfristig möglich werden. 